\title{
A REMARK ON CARLESON'S CHARACTERIZATION OF BMO
}

\section{AKIHITO UCHIYAMA}

\begin{abstract}
L. Carleson showed that if $\varphi \in$ BMO $\left(R^{n}\right)$ and supp $\varphi$ is compact, then $\varphi$ can be written in the form $\varphi(x)=\sum_{k-1}^{\infty} \int P_{t_{k}(y)}(x-y) b_{k}(y) d y+b_{0}(x)$ where $\sum_{k-0}^{\infty}\left\|b_{k}\right\|_{\infty}<C\|\varphi\|_{B M O}, t_{k}(y)>0$ and $P_{t}(x)=C_{n} t\left(|x|^{2}+t^{2}\right)^{-(n+1) / 2}$ is the Poisson kernel. We show that we can take $b_{2}=b_{3}=\cdots=0$. This can be generalized on the space of homogeneous type with certain assumptions.
\end{abstract}

1. Introduction. All functions considered are real valued measurable functions. For $\varphi \in L_{\mathrm{loc}}^{1}\left(R^{n}\right)$ we define

$$
\|\varphi\|_{\mathrm{BMO}}=\sup _{B} \inf _{c \in R}|B|^{-1} \int_{B}|\varphi(x)-c| d x
$$

where the supremum is taken over all balls $B$ and $|B|$ denotes the Lebesgue measure of $B$. And we define $\operatorname{BMO}\left(R^{n}\right)=\left\{\varphi:\|\varphi\|_{\text {BMO }}<\infty\right\}$. In [3], Fefferman and Stein showed that $\mathrm{BMO}\left(R^{n}\right)$ is the dual space of $H^{1}\left(R^{n}\right)$, where

$$
\begin{aligned}
\|g\|_{H^{1}} & =\|g\|_{L^{1}}+\sum_{j=1}^{n}\left\|R_{j}(g)\right\|_{L^{1}} \\
R_{j}(g) & =F^{-1}\left(\xi_{j}\left(\sum_{k=1}^{n} \xi_{k}^{2}\right)^{-1 / 2}(F(g))(\xi)\right)
\end{aligned}
$$

and $F$ denotes the Fourier transform.

Recently L. Carleson [1] showed a new characterization of BMO which gives another proof of the duality between $H^{1}$ and BMO, that is,

Theorem A. Let $h \in C^{1}\left(R^{n}\right)$ and assume that $h(x),|\nabla h(x)|<(1+|x|)^{-n-1}$, $h(x) \geqslant 0, \int h(x) d x=1$ and $h(x)$ is even. Let $\|\varphi\|_{\mathrm{BMO}}<1$ and supp $\varphi \subset B_{1}$, where $B_{t}=\left\{x \in R^{n}:|x|<t\right\}$. Then there exists a positive number $M$, only depending on $n$, a sequence of functions $b_{k}(x)$ such that $\sum_{k=0}^{\infty}\left\|b_{k}\right\|_{\infty}<M$ and $t_{k}(y)>0$ such that

$$
\varphi(x)=\sum_{k \in N} \int h_{t_{k}(y)}(x-y) b_{k}(y) d y+b_{0}(x)
$$

where $h_{t}(x)=t^{-n} h(x / t)$ and $N=\{1,2,3, \ldots\}$.

Carleson's proof showed that we can take

$$
b_{2}=b_{3}=\cdots=0
$$

Received by the editors November 7, 1978.

AMS (MOS) subject classifications (1970). Primary 46E99; Secondary 46E30.

Key words and phrases. BMO, Poisson kernel, space of homogeneous type. 
provided that supp $h \subset B_{1}$. But, in Fourier analysis, important cases occur when the support of $h$ is not bounded, for example, the Poisson kernel

$$
P_{t}(x)=C_{n} t /\left(|x|^{2}+t^{2}\right)^{(n+1) / 2} \text {. }
$$

Now, we show (1) is possible in general.

THEOREM 1. Let $\varphi$ and $h$ be as in Theorem A. Then there exists a positive number $M$, depending only on $n$, bounded functions $b_{0}(x), b_{1}(x)$ and a positive function $t(y)$ such that

$$
\begin{gathered}
\left\|b_{0}\right\|_{\infty}+\left\|b_{1}\right\|_{\infty}<M \\
\varphi(x)=\int h_{t(y)}(x-y) b_{1}(y) d y+b_{0}(x) .
\end{gathered}
$$

Concerning the duality between $H^{1}$ and $\mathrm{BMO}$, our modification does not give more information than does Carleson's result, but it means that there exists a Carleson measure $\nu$ of a special type such that

$$
\varphi(x)=\int_{R^{n} \times(0, \infty)} P_{t}(x-y) d \nu(y, t)+b_{0}(x) .
$$

(For the definition of the Carleson measure see [6, p. 222].)

In $\$ 3$, we extend this result to the space of homogeneous type with certain geometric assumptions, in particular to $\operatorname{BMO}\left(\partial B^{n}\right)$.

The letters $C$ and $c$ will be used to denote various constants and the various uses of them do not all denote the same constant.

2. The main theory. In the proof of Theorem A, Carleson showed

THEOREM B. Let $\varphi$ and $h$ be as in Theorem A. Then there exist functions $\left\{\beta_{1 k}(x)\right\}_{k \in N}, \beta_{1}(x)$ and $\varphi^{(1)}(x)$ such that supp $\beta_{1 k} \subset B_{c}$, supp $\beta_{1 k} \cap \operatorname{supp} \beta_{1 h}=\varnothing$ $(k \neq h),\left\|\beta_{1 k}\right\|_{\infty}<C,\left\|\beta_{1}\right\|_{\infty}<C, \operatorname{supp} \varphi^{(1)} \subset B_{c},\left\|\varphi^{(1)}\right\|_{\mathrm{BMO}}<1 / 2, r_{k}=C 2^{-C k}$ and

$$
\varphi(x)=\sum_{k \in N} h_{r_{k}} * \beta_{1 k}(x)+\beta_{1}(x)+\varphi^{(1)}(x)
$$

Next he applied Theorem B to $2 \varphi^{(1)}\left(2^{C k_{0}} x\right)$, where $k_{0}$ is sufficiently large. Then

$$
2 \varphi^{(1)}\left(2^{C k_{0}} x\right)=\sum_{k \in N} h_{r_{k}} * \beta_{2 k}^{\prime}(x)+\beta_{2}^{\prime}(x)+\varphi^{(2) \prime}(x) .
$$

Changing the scale and multiplying by $\frac{1}{2}$,

$$
\varphi^{(1)}(x)=\sum_{k \in Z, k>-k_{0}} h_{r_{k}} * \beta_{2 k}(x)+\beta_{2}(x)+\varphi^{(2)}(x)
$$

where $Z=\{0, \pm 1, \pm 2, \ldots\}$. Repeating this argument, we get

$$
\varphi(x)=\sum_{j \in N}\left(\sum_{k \in Z} h_{r_{k}} * \beta_{j k}(x)+\beta_{j}(x)\right)
$$

where $\left\|\beta_{j}\right\|_{\infty} \leqslant 2^{-j+1} C,\left\|\beta_{j k}\right\|_{\infty} \leqslant 2^{-j+1} C$, supp $\beta_{j k} \subset B_{c}$, $\left\{\operatorname{supp} \beta_{j k}\right\}_{k \in Z}$ are mutually disjoint, $\beta_{j k}(x) \equiv 0$ for $k \leqslant-j k_{0}$. 
This implies Theorem A. In the above we note that

$$
\begin{aligned}
\left\|\sum_{j \in N} \sum_{k=-\infty}^{0} h_{r_{k}} *\left|\beta_{j k}\right|\right\|_{\infty}<C, \\
\quad \sum_{j \in N} \sum_{k \in N} h_{r_{k}} *\left|\beta_{j k}\right|(x)<\infty \text { for a.e. } x .
\end{aligned}
$$

Thus changing the order of the summation, we get

$$
\varphi(x)=\sum_{k \in N} h_{r_{k}} *\left(\sum_{j \in N} \beta_{j k}\right)(x)+\sum_{j \in N} \sum_{k=-\infty}^{0} h_{r_{k}} * \beta_{j k}(x)+\sum_{j \in N} \beta_{j}(x) .
$$

Since the last two terms are bounded, we get

Corollary. Assume that $\|\varphi\|_{\mathrm{BMO}}<C$ and $\operatorname{supp} \varphi \subset B_{1}$. Then there exist bounded functions $\left\{d_{k}(x)\right\}_{k \in N}, d(x)$ and positive numbers $\left\{r_{k}\right\}_{k \in N}$ such that

$$
\begin{gathered}
\left\|\sum_{k \in N}\left|d_{k}\right|\right\|_{\infty}<1, \\
\|d\|_{\infty}<1, \\
\varphi(x)=\sum_{k \in N} h_{r_{k}} * d_{k}(x)+d(x) .
\end{gathered}
$$

To prove Theorem 1, we need the above corollary and the following

LeMma. Let $(\Omega, F, \mu)$ be a $\sigma$-finite measure space with no atoms. Let $\left\{F_{k}\right\}_{k \in N}$ be a sequence of nondecreasing atomic sub- $\sigma$-fields of $F$. Let $A_{k}$ be the set of the atoms of $F_{k}$. Let $\left\{u_{k}(x)\right\}_{k \in N}$ be a sequence of nonnegative functions such that $\left\|\Sigma_{k \in N} u_{k}\right\|_{\infty}<$ 1. Then there exist mutually disjoint $F$-measurable sets $\left\{H_{k}\right\}_{k \in N}$ such that

$$
\mu\left(H_{k} \cap B\right)=\int_{B} u_{k}(x) d \mu(x)
$$

for any $k \in N$ and $B \in A_{k}$.

Proof of Theorem 1. For simplicity we consider the case $n=1$. We may assume that $\|\varphi\|_{\text {BMO }}$ is sufficiently small. By the corollary, we get bounded functions $\left\{d_{k}(x)\right\}_{k \in N}, d(x)$ and positive numbers $\left\{r_{k}\right\}_{k \in N}$ with (4), (5) and (6). Let $\left\{\varepsilon_{k}\right\}_{k \in N}$ be positive numbers such that $2^{-k} r_{k}>\varepsilon_{k}$ and $\varepsilon_{k} / \varepsilon_{k+1} \in N$ for any $k \in N$. Let $\left\{F_{k}\right\}_{k \in N}$ be the atomic sub- $\sigma$-fields such that $A_{k}=\left\{\left[j \varepsilon_{k},(j+1) \varepsilon_{k}\right)\right.$ : $j \in Z\}$, where $A_{k}$ is as in the lemma. Set $u_{k}(x)=\left|d_{k}(x)\right|$. Then, by (4) and the lemma, we get mutually disjoint measurable sets $\left\{H_{k}\right\}_{k \in N}$ with (7). If $x \in H_{k}$, we put

$$
f_{k}(x)=\left|H_{k} \cap\left[j \varepsilon_{k},(j+1) \varepsilon_{k}\right)\right|^{-1} \int_{j \varepsilon_{k}}^{(j+1) \varepsilon_{k}} d_{k}(y) d y
$$

where $x \in\left[j \varepsilon_{k},(j+1) \varepsilon_{k}\right)$. If $x \notin H_{k}$, we put $f_{k}(x)=0$. Then, by (7), $\left\|f_{k}\right\|_{\infty}<1$. 
And

$$
\begin{aligned}
\mid \int_{R} h_{r_{k}}(x- & y)\left(d_{k}(y)-f_{k}(y)\right) d y \mid \\
& <\sum_{j \in Z} \int_{j \varepsilon_{k}}^{(j+1) \varepsilon_{k}}\left|h_{r_{k}}(x-y)-h_{r_{k}}\left(x-j \varepsilon_{k}\right)\right|\left|d_{k}(y)-f_{k}(y)\right| d y \\
& \leqslant\left(\left\|d_{k}\right\|_{\infty}+\left\|f_{k}\right\|_{\infty}\right) C\left(\varepsilon_{k} / r_{k}\right) \int_{R}\left(1+|x-y| / r_{k}\right)^{-2} r_{k}^{-1} d y<C 2^{-k}
\end{aligned}
$$

Thus, by (6), $\varphi(x)=\Sigma_{k \in N} h_{r_{k}} * f_{k}(x)+\Sigma_{k \in N} h_{r_{k}} *\left(d_{k}-f_{k}\right)(x)+d(x)$ where $\left\|\Sigma_{k \in N} h_{r_{k}} *\left(d_{k}-f_{k}\right)+d\right\|_{\infty}<C,\left\|f_{k}\right\|<1$ and $\left\{\operatorname{supp} f_{k}\right\}_{k \in N}$ are mutually disjoint. Set $b_{1}(x)=f_{k}(x)$ on $H_{k}$ for $k \in N, b_{1}(x)=0$ on $\left(\cup H_{k}\right)^{c}, b_{0}(x)=$ $\Sigma_{k \in N} h_{r_{k}} *\left(d_{k}-f_{k}\right)(x)+d(x), t(y)=r_{k}$ on $H_{k}$ for $k \in N, t(y)=1$ on $\left(\cup H_{k}\right)^{c}$. Then the conditions (2) and (3) are satisfied. Q.E.D.

3. BMO on the space of homogeneous type. Following [2], we consider BMO on the space of homogeneous type. Let $X$ be a topological space with a Borel measure $\mu$, a quasi-distance $\rho$ and functions $\left\{K_{r}\right\}_{0<r<\mu(X)}$ on $X \times X$ satisfying the following properties: there exist positive numbers $C_{1}, C_{2}$ and $\alpha$ such that

(a) $\rho(x, y)=\rho(y, x)>0$,

(b) $\rho(x, y)=0$ iff $x=y$,

(c) $\rho(x, z) \leqslant C_{1}(\rho(x, y)+\rho(y, z))$,

(d) $C_{2} r \leqslant \mu\left(B_{r}(x)\right) \leqslant C_{1} r$ where $B_{r}(x)=\{y \in X: \rho(x, y)<r\}$ and $0<r<$ $\mu(X)$,

(e) $\left\{B_{r}(x)\right\}_{r>0}$ form a basis of open neighbourhoods of $x$,

(f) $|\rho(x, y)-\rho(x, z)| / \rho(x, y)<C_{1}(\rho(y, z) / \rho(x, y))^{\alpha}$ if $\rho(y, z)<C_{2} \rho(x, y)$,

(g) $K_{r}(x, y) \geqslant 0$,

(h) $\inf \left\{\int_{B_{r}(z)} K_{r / C_{1}}(x, y) d \mu(y): r>0, z \in X, B_{r}(z) \ni x\right\}>C_{2}$,

(i) $\sup _{x, r}\left|\int_{X} K_{r}(x, y) d \mu(y)-1\right|<1 / 10$,

(j) $K_{r}(x, y)<C_{1}(1+\rho(x, y) / r)^{-1-\alpha} / r$,

(k) $\max \left(\left|K_{r}(x, y)-K_{r}(z, y)\right|, \quad\left|K_{r}(y, x)-K_{r}(y, z)\right|\right)<C_{1}(\rho(x, z) / r)^{\alpha}(1+$ $\rho(x, y) / r)^{-1-\alpha} / r$ if $\rho(x, z) \leqslant C_{2} r$.

For $f \in L_{\text {loc }}^{1}(X)$ (i.e. $\left.f\right|_{B} \in L^{1}(B)$ for all $\left.B=B_{r}(x)\right)$ we define

$$
\|f\|_{\text {BMO }(X)}=\sup _{B} \inf _{c \in R}|B|^{-1} \int_{B}|f(x)-c| d \mu(x)
$$

if $\mu(X)=\infty$, where the supremum is taken over all $B=B_{r}(x)(r>0, x \in X)$. If $\mu(X)<\infty$, we define

$$
\|f\|_{\text {BMO }(X)}=\sup _{B} \inf _{c \in R}|B|^{-1} \int_{B}|f(x)-c| d \mu(x)+\left|\int_{X} f(x) d \mu(x)\right| .
$$

Then we can extend Theorem 1 to this space.

TheOREM 2. Let $\|\varphi\|_{\mathrm{BMO}(X)}<1$ and supp $\varphi \subset B_{r_{0}}\left(x_{0}\right)$ for some $r_{0}>0$ and $x_{0} \in$ $X$. Then there exist bounded functions $b_{0}(x)$ and $b_{1}(x)$ such that $\left\|b_{0}\right\|_{\infty}+\left\|b_{1}\right\|_{\infty}<M$ and a positive function $t(y)$ such that the range of $t$ is a discrete subset of $R^{+} \backslash\{0\}$ 
and that

$$
\varphi(x)=\int K_{t(y)}(x, y) b_{1}(y) d \mu(y)+b_{0}(x)
$$

where $M$ is independent of $\varphi, r_{0}$ and $x_{0}$.

The proof is the same as with Theorem 1 and we will omit it. [This kind of generalization is announced in [2] under the assumption $K_{r}(x, y)=0$ if $\rho(x, y)>$ r.]

ExAmple. $\partial B^{n}$ denotes $\left\{z=\left(z_{1}, \ldots, z_{n}\right) \in C^{n}: \sum_{k=1}^{n}\left|z_{k}\right|^{2}=1\right\}$. On $\partial B^{n}$ we define $\rho(z, w)=\left|1-\sum_{k=1}^{n} z_{k} \bar{w}_{k}\right|^{n}$ and $K_{r}(z, w)=P\left(\left(1-r^{1 / n}\right) z, w\right)$, where $P(\zeta, z)$ $=C_{n}\left(1-|\zeta|^{2}\right)^{n} /|1-\zeta \cdot \bar{z}|^{2 n}$ that is the Poisson-Szegö kernel. Then, by using the Lebesgue surface measure $d \sigma$, we obtain a space of homogeneous type with (a)-(k). Thus, by Theorem 2 , for any $\varphi \in \operatorname{BMO}\left(\partial B^{n}\right)$, there exist bounded functions $b_{0}(z), b_{1}(z)$ and $t(z)$ such that $1 / 2<t(z)<1,\left\|b_{0}\right\|_{\infty}+\left\|b_{1}\right\|_{\infty}<M\|\varphi\|_{\mathrm{BMO}}$ and

$$
\varphi(z)=\int_{\partial B^{n}} P(t(w) w, z) b_{1}(w) d \sigma(w)+b_{0}(z) .
$$

4. Proof of lemma. In the following $k, p, q \in N$. We may assume

$$
\begin{gathered}
\sup \left\{\mu(B): B \in \bigcup_{k \in N} A_{k}\right\}<\infty, \\
\lim _{k \rightarrow \infty} \sup \left\{\mu(B): B \in A_{k}\right\}=0 .
\end{gathered}
$$

Let $A_{1}=\{B(1), B(2), \ldots\}$. For each $B(p)$ let $\{B(p, 1), B(p, 2), \ldots\}$ be the elements of $A_{2}$ contained in $B(p)$. In this way $A_{k}(k \in N)$ can be indexed inductively as follows.

$$
\begin{gathered}
A_{k}=\left\{B\left(p_{1}, \ldots, p_{k}\right)\right\}_{1<p_{q}<N_{q}\left(p_{1}, \ldots, p_{q-1}\right)} \quad(q=1, \ldots, k), \\
N_{q}\left(p_{1}, \ldots, p_{q-1}\right) \in\{2,3,4, \ldots, \infty\}, \quad B\left(p_{1}, \ldots, p_{k}\right) \subset B\left(p_{1}, \ldots, p_{q}\right) \\
\quad \text { if } q \in\{1, \ldots, k\} .
\end{gathered}
$$

Set $I=\left\{\left(p_{1}, \ldots, p_{k}\right): k \in N, 1<p_{q}<N_{q}\left(p_{1}, \ldots, p_{q-1}\right)\right.$ for $\left.q=1, \ldots, k\right\}$, $\left|\left(p_{1}, \ldots, p_{k}\right)\right|=k$ and $\left(p_{1}, \ldots, p_{k}\right)(q)=p_{q}$ for $q=1,2, \ldots, k$. In the following (i) and $(j)$ stand for elements of $I .(i) \prec(j)$ means $|(i)|>|(j)|$ and $(i)(k)=(j)(k)$ for $k=1, \ldots,|(j)|$.

For each $(i) \in I$ and $a \in\left[0, \mu(B(i))-\int_{B(i)} \sum_{k=|(i)|+1}^{\infty} u_{k} d \mu\right)$, we set $m=|(i)|$,

$$
\begin{aligned}
M_{m+1}((i), a)= & \inf \left\{p: \sum_{k=1}^{p}\left(\mu(B(i, k))-\int_{B(i, k)} \sum_{q=m+1}^{\infty} u_{q} d \mu\right)>a\right\} \\
a_{m+1}((i), a)= & a-\sum_{k=1}^{M_{m+1}-1}\left(\mu(B(i, k))-\int_{B(i, k)} \sum_{q=m+1}^{\infty} u_{q} d \mu\right) \\
& +\int_{B\left(i, M_{m+1}\right)} u_{m+1} d \mu \\
G_{m+1}((i), a)= & \bigcup_{k=1}^{M_{m+1}-1} B(i, k) .
\end{aligned}
$$


By the condition on $a, M_{m+1}$ is well defined. Since, by the definition of $M_{m+1}$,

$$
a_{m+1} \in\left[0, \mu\left(B\left(i, M_{m+1}\right)\right)-\int_{B\left(i, M_{m+1}\right)} \sum_{k=m+2}^{\infty} u_{k} d \mu\right),
$$

we can define $M_{m+k}, a_{m+k}$ and $G_{m+k}$ inductively as follows:

$$
\begin{gathered}
M_{m+k}((i), a)=M_{m+k}\left(\left(i, M_{m+1}\right), a_{m+1}((i), a)\right), \\
a_{m+k}((i), a)=a_{m+k}\left(\left(i, M_{m+1}\right), a_{m+1}((i), a)\right), \\
G_{m+k}((i), a)=G_{m+k}\left(\left(i, M_{m+1}\right), a_{m+1}((i), a)\right) .
\end{gathered}
$$

Set

$$
\begin{aligned}
E((i), a) & =\bigcup_{k=1}^{\infty} G_{|(i)|+k}((i), a), \\
F(i) & =E\left((i), \int_{B(i)} u_{|(i)|} d \mu\right), \\
J_{k}(i) & =\left\{B \in A_{k}: \mu(B \cap F(i))>0\right\} \text { for } k>|(i)| .
\end{aligned}
$$

Then

(9) $F(i) \subset B(i)$.

(10) $\mu(F(i))=\sum_{k=|(i)|}^{\infty} \Sigma_{B \in J_{k}(i)} \int_{B} u_{k} d \mu$.

(11) If $\mu(F(i) \cap B(j))>0$ and $(i)>(j)$, then $F(i) \supset F(j)$.

(12) If $\mu(F(i) \cap F(j))=0$, then $J_{k}(i) \cap J_{k}(j)=\varnothing$ for any $k>\max (|(i)|,|(j)|)$.

(13) If $\int_{B(i)} u_{|(i)|} d \mu>0$, then $\mu(F(i))>0$.

Set $K(i)=F(i) \backslash \cup_{(j)<(i)} F(j)$. By (9) and (11), there exists a subset $L(i)$ of $I$ with the properties: (a) $(j) \prec(i)$ and $F(j) \subset F(i)$ for any $(j) \in L(i)$, (b) $\{F(j)\}_{(j) \in L(i)}$ are mutually disjoint, (c) if $(j) \prec(i)$ and $F(j) \subset F(i)$, then there exists $\left(j_{0}\right) \in L(i)$ such that $F(j) \subset F\left(j_{0}\right)$. Thus by (10)-(12) and the above property of $L(i)$, we have

$$
\begin{aligned}
\mu(K(i)) & =\mu(F(i))-\sum_{(j) \in L(i)} \mu(F(j)) \\
& =\sum_{k=|(i)|}^{\infty} \sum_{B \in J_{k}(i)} \int_{B} u_{k} d \mu-\sum_{(j) \in L(i)} \sum_{k=|(j)|}^{\infty} \sum_{B \in J_{k}(j)} \int_{B} u_{k} d \mu \\
& =\int_{B(i)} u_{|(i)|} d \mu .
\end{aligned}
$$

Set $H_{k}=\cup_{(i) \in I,|(i)|-k} K(i)$, then $\left\{H_{k}\right\}_{k=1}^{\infty}$ has the required property.

Lastly we prove (9)-(13). (9) and (10) are obvious. (13) follows from the assumption (8). Note that

$$
E\left((i), a_{1}\right) \subset E\left((i), a_{2}\right)
$$


provided $0 \leqslant a_{1} \leqslant a_{2}<\mu(B(i))-\int_{B(i)} \sum_{k=|(i)|+1}^{\infty} u_{k} d \mu$ and note that

$$
\begin{aligned}
E((i), a) & =\left(\bigcup_{k=|(i)|+1}^{p} G_{k}((i), a)\right) \cup\left(\bigcup_{k=p+1}^{\infty} G_{k}((i), a)\right) \\
& \supset \bigcup_{k=p+1}^{\infty} G_{k}((i), a)=E\left(\left(i, M_{|(i)|+1}((i), a), \ldots, M_{p}((i), a)\right), a_{p}((i), a)\right)
\end{aligned}
$$

provided $p>|(i)|$. From (14), (15) and

$$
a_{p}((i), a) \geqslant \int_{B\left(i, M_{|(i)|+1}((i), a), \ldots, M_{p}((i), a)\right)} u_{p} d \mu,
$$

(11) and(12) follow easily. Q.E.D.

Acknowledgement. I would like to thank Professor Richard R. Goldberg for valuable information.

\section{REFERENCES}

1. L. Carleson, Two remarks on $H^{1}$ and BMO, Advances in Math. 22 (1976), 269-277.

2. R. Coifman and G. Weiss, Extensions of Hardy spaces and their use in analysis, Bull. Amer. Math. Soc. 83 (1977), 569-645.

3. C. Fefferman and E. M. Stein, $H^{p}$ spaces of several variables, Acta Math. 129 (1972), 137-193.

4. F. John and L. Nirenberg, On functions of bounded mean oscillation, Comm. Pure Appl. Math. 14 (1961), 415-426.

5. E. M. Stein, Boundary behavior of holomorphic functions of several complex variables, Princeton Univ. Press, Princeton, N. J., 1972.

6. N. Th. Varopoulos, BMO functions and the $\bar{\partial}$-equation, Pacific J. Math. 71 (1977), 221-273.

7. R. Coifman and R. Rochberg, Another characterization of BMO (preprint).

Department of Mathematics, College of General Education, Tôhoku University, Kawauch, SENDAI, JAPAN 